\title{
Compose Quotient Ring Sequences with Walsh's Sequences and M-Sequences
}

\author{
Ahmad Hamza Al Cheikha \\ Department of Mathematical Science, College of Arts-Science and Education, Ahlia University, Manama, Bahrain \\ Email address: \\ alcheikhaa@yahoo.com \\ To cite this article: \\ Ahmad Hamza Al Cheikha. Compose Quotient Ring Sequences with Walsh's Sequences and M-Sequences. International Journal of \\ Theoretical and Applied Mathematics. Vol. 5, No. 1, 2019, pp. 10-20. doi: 10.11648/j.ijtam.20190501.12
}

Received: December 7, 2018; Accepted: April 4, 2019; Published: May 6, 2019

\begin{abstract}
Quotient ring sequences are completely new orthogonal sets without coders and decoders to the moment but Walsh sequences of the order $2^{k}, k$ positive integer, and M-Sequences with zero sequence form additive groups, Except the zero sequences, Walsh sequences, and M-Sequences formed orthogonal sets and used widely in the forward links and inverse links of communication channels for mixing and sifting information as in the systems CDMA and other channels. The current paper studied the orthogonal sets (which are also with the corresponding null sequence additive groups) generated through compose quotient ring sequences with self, Compose quotient ring sequences with the best and very important sequences Walsh sequences and M-sequences and by inverse for getting these new orthogonal sets or sequences with longer lengths and longer minimum distances in order to increase the confidentiality of information and increase the possibility of correcting mistakes in the communication channels.
\end{abstract}

Keywords: Quotient ring Sequences, Walsh Sequences, M-sequences, Coefficient of Correlation, Code, Orthogonal Sequences, Additive group, Span

\section{Introduction}

\subsection{Orthogonal Quotient Ring Sequences}

We can get quotient ring sequences from the Multiplication table of quotient rings $Z_{p^{m}}=Z /\left(p^{m} Z\right)$, where $\mathrm{Z}$ is the Integers and $\mathrm{p}$ is a prime number, deleting the rows which have index multiple of $p$, replacing each event number by " 0 " and each odd number by " 1 ", and choosing one of the subsets of binary rows which each row in it contains $\left(p^{m}+1\right) / 2$ of “ $0 . \mathrm{s}$ " and $\left(p^{m}-1\right) / 2$ of "1.s" (Length each row is $p^{m}$ ) and each subset has, without zero row, a biggest orthogonal span (its size is u, where $u=\sum_{i=1}^{m+1}\left(\begin{array}{l}m+1 \\ i\end{array}\right)$ ). These biggest orthogonal span, we say, $\mathrm{Q}=\left\{q_{1}, q_{2}, \ldots, q_{u}\right\}$, with zero row $q_{0}=r_{0}$ forms an additive subgroup in the vector space $2^{\left(p^{m}\right)}$. The number of these subsets is at most $\left(\begin{array}{c}p^{m}-p^{m-1} \\ m+1\end{array}\right)$.
For $p=2$ we get Walsh sequences. [3]

\subsection{Walsh Sequence}

Walsh sequences are binary sets with $2^{k}$ of rows (or sequences), except the zero row, each set is orthogonal, the length of each row is $2^{k}$ and contains $2^{k-1}$ of " $0 . \mathrm{s}$ " and the same number of "1.s", and forms an additive group with the zero row where the addition performed by $\bmod 2$, also they are known under the name Walsh functions.

The Walsh functions can be generated by any of the following methods:

(1) Using Rademacher functions.

(2) Using Hadamard matrices. [4]

(3) Exploiting the symmetry properties of Walsh functions. [5]:

(4) Using division ring under $2^{k}$ addition. $[6,7]$

\subsection{Binary M-Sequences: M-Linear Recurring Sequences}

Let $k$ be a positive integer and $\lambda, \lambda_{0}, \lambda_{1}, \ldots, \lambda_{k-1}$ are elements in the field $F_{2}$ then the sequence $z_{0}, z_{1}, \ldots$ is called non homogeneous linear recurring sequence of order $k$ iff: 


$$
\begin{aligned}
& z_{n+k}=\lambda_{k-1} z_{n+k-1}+\lambda_{k-2} z_{n+k-2}+\ldots \\
& +\lambda_{0} z_{n}+\lambda, \lambda_{i} \in F_{2}, i=0,1, \ldots, k-1 ; \\
& \text { or } \quad z_{n+k}=\sum_{i=0}^{k-1} \lambda_{i} z_{n+i}+\lambda
\end{aligned}
$$

The elements $z_{0}, z_{1}, \ldots, z_{k-1}$ are called the initial values (or the vector $\left(z_{0}, z_{1}, \ldots, z_{k-1}\right)$ is called the initial vector).

If $\lambda=0$ then the sequence $z_{0}, z_{1}, \ldots$ is called homogeneous linear recurring sequence (H. L. R. S.), except the zero initial vector, and the polynomial

$$
f(x)=x^{k}+\lambda_{k-1} x^{k-1}+\ldots+\lambda_{1} x+\lambda_{0}
$$

Is called the characteristic polynomial. In this study, we are limited to $\lambda_{0}=1$. $[8,9]$.

Orthogonal quotient rings sequences are good and important sequences absolutely new, published one month ago, linear, suitable and sufficient lengths and minimum distances, to this moment there is no coders and decoders for them.

Thus, sequence generated showed increased secrecy and increased possibility of correcting error in communication channel because it exhibited bigger length and the bigger minimum distance.

\section{Research Method and Materials}

Definition 1.The Ultimately Periodic Sequence $z_{0}, z_{1}, \ldots$. with the smallest period $r$ is called a periodic iff:

$$
z_{n+r}=z_{n ;}, n=0,1, \ldots[10]
$$

Definition 2. The complement of the binary vector $X=\left(x_{1}, x_{2}, \ldots, x_{n}\right)$ is the vector

$$
\bar{X}=\left(\overline{x_{1}}, \overline{x_{2}}, \ldots, \overline{x_{n}}\right)
$$

When;

$$
\overline{x_{i}}=\left\{\begin{array}{lll}
1 & \text { if } & x_{i}=0 \\
0 & \text { if } & x_{i}=1
\end{array} .[11]\right.
$$

Definition 3. Suppose $\quad x=\left(x_{0}, x_{1}, \ldots, x_{n-1}\right) \quad$ and $y=\left(y_{0}, y_{1}, \ldots, y_{n-1}\right)$ are vectors of length $n$ on $G F(2)=$

$F_{2}=\{0,1\}$. The coefficient of correlations function of $x$ and $y$,

Denoted by $R_{x, y}$, is:

$$
R_{x, y}=\sum_{i=0}^{n-1}(-1)^{x_{i}+y_{i}}
$$

Definition 4. Any Periodic Sequence $z_{0}, z_{1}, \ldots$ over $F_{2}$ with prime characteristic polynomial is an orthogonal cyclic code and ideal auto correlation. [13,14]

Definition 5. Suppose $G$ is a set of binary vectors of length $n$ :

$$
G=\left\{X ; X=\left(x_{0}, x_{1}, \ldots, x_{n-1}\right), x_{i} \in F_{2}, i=\{0, \ldots, n-1\}\right\}
$$

Let $1^{*}=-1$ and $0^{*}=1$. The set $G$ is said to be orthogonal if the following two conditions are satisfied:

$$
\forall X \in G, \sum_{i=0}^{n-1} x_{i}^{*} \in\{-1,0,1\} \quad \text { or } \quad\left|R_{x, 0}\right| \leq 1
$$

$\forall X, Y \in G$ and $X \neq Y, \sum_{i=0}^{n-1} x_{i}^{*} y_{i}^{*} \in\{-1,0,1\}$, or $\ldots\left|R_{x, y}\right| \leq 1$

That is, the absolute value of "the number of agreements minus the number of disagreements" is equal to or less than 1. [15]

Definition 6. Hamming distance $d(x, y)$ : The Hamming distance between the binary vectors $x=\left(x_{0}, \ldots, x_{n-1}\right)$ and

$y=\left(y_{0}, y_{1}, \ldots, y_{n-1}\right)$ is the number of the disagreements of the corresponding components of $x$ and $y$. [16]

Definition 7. If $C$ is a set of binary sequences and $\omega$ is any binary vector then $C(\omega)=\left\{x_{i}(\omega): x_{i} \in C\right\}$ We replace each " 1 " in $x_{i}$ by $\omega$ and each " 0 " in $x_{i}$ by $\bar{\omega}$.

Corollary 1 . If in the binary vector $x$ : the number of " $1 . s$ " and the number of " $0 . \mathrm{s}$ " are $m_{1}$ and $m_{2}$ respectively, and in the Binary vector $w$ the number of' $1 . \mathrm{s}$ " and the number of " $0 . \mathrm{s}$ " are $n_{1}$ and $n_{2}$ respectively then in the binary vector $x(w)$.

The number of "1.s" and the number of " $0 . s "$ are $m_{1} n_{1}+m_{2} n_{2}$ and $m_{1} n_{2}+m_{2} n_{1}$ respectively. [17]

Theorem 2 .

If $a_{0}, a_{1}, \ldots$ is a homogeneous linear recurring sequence of order $k$ in $F_{2}$, satisfies (1) then this sequence is periodic.

If the characteristic polynomial $f(x)$ of the sequence is primitive then the period of the Sequence is $2^{k}-1$, and this sequence is called $\mathrm{M}$-sequence and each of these sequences contains $2^{k-1}$ of " 1 "s and $2^{k-1}-1$ of " 0 "s. $[18,19]$

\section{Results and Discussions (Findings)}

\subsection{Compose Quotient Ring Sequences with Other Quotient Ring Sequences}

Suppose $Q_{1}=\left\{q_{1}, q_{2}, \ldots, q_{u_{1}}\right\}$ and $Q_{2}=\left\{q_{1}^{\prime}, q_{2}^{\prime}, \ldots, q_{u_{2}}^{\prime}\right\}$ are two orthogonal quotient rings sequences generated from binary representation of $Z_{p_{1}^{m}}, Z_{p_{2}^{n}}$ respectively, then in $Q_{1}\left(Q_{2}\right)$ : 
Table 1. The numbers of "1.s" and "0.s" in each sequence of $Q_{1}$ and $Q_{2}$

\begin{tabular}{llll}
\hline $\boldsymbol{Q}_{1}$ & & $\boldsymbol{Q}_{2}$ & Number of "0.s" \\
\hline Number of "1.s" & Number of " $0 . \mathbf{s}$ " & Number of "1.s" & $\frac{p_{2}^{n}+1}{2}$ \\
\hline$\frac{p_{1}^{m}-1}{2}$ & $\frac{p_{1}^{m}+1}{2}$ & $\frac{p_{2}^{n}-1}{2}$ & \\
\hline
\end{tabular}

A) For $q_{k}^{\prime} \in Q_{2}$ we define the set:

$A_{k}=Q_{1}\left(q_{k}^{\prime}\right)=\left\{a_{i}=q_{i}\left(q_{k}^{\prime}\right), q_{i} \in Q_{1}\right\}$ then:

(1) The number of " $1 . \mathrm{s}$ " in $a_{i}$ is:

$$
\left(\frac{p_{1}^{m}-1}{2}\right)\left(\frac{p_{2}^{n}-1}{2}\right)+\left(\frac{p_{1}^{m}+1}{2}\right)\left(\frac{p_{2}^{n}+1}{2}\right)=\frac{p_{1}^{m} p_{2}^{n}+1}{2}
$$

(2) The number of " $0 . \mathrm{s}$ " in $a_{i}$ is:

$\left(\frac{p_{1}^{m}-1}{2}\right)\left(\frac{p_{2}^{n}+1}{2}\right)+\left(\frac{p_{1}^{m}+1}{2}\right)\left(\frac{p_{2}^{n}-1}{2}\right)=\frac{p_{1}^{m} p_{2}^{n}-1}{2}$

(3) The difference between the number of " 1 "s and the number of " 0 "s is one

b) For $a_{i}, a_{j} \in A_{k}$ and $i \neq j, a_{i}+a_{j}=q_{i}\left(q_{k}^{\prime}\right)+q_{j}\left(q_{k}^{\prime}\right)$

$a_{i}+a_{j}=\left(q_{i}+q_{j}\right)(11 \ldots 1)_{p_{2}^{n}}$ and $a_{i}+a_{j} \neq\left(q_{i}+q_{j}\right)\left(q_{k}^{\prime}\right)$

(4) The number of " $1 . \mathrm{s}$ " in $a_{i}+a_{j}$ is $\frac{p_{1}^{m}-1}{2}\left(p_{2}^{n}\right)$, the number of " $0 . \mathrm{s}$ " in $a_{i}+a_{j}$ is $\frac{p_{1}^{m}+1}{2}\left(p_{2}^{n}\right)$

(5) The difference between the number of " $0 . s$ " and the number of " $1 . \mathrm{s}$ " is $p_{2}^{n}$

Thus, $A_{k}$ is not an orthogonal set and $Q_{1}\left(Q_{2}\right)$ are not orthogonal sets.

c) By symmetric property $Q_{2}\left(Q_{1}\right)$ are not orthogonal sets.

d) By the same way for $q_{i}, q_{j} \in Q_{1}$ and $i \neq j$, then $\bar{q}_{i}, \bar{q}_{j}$ satisfies the first orthogonal condition but: $\bar{q}_{i}\left(q_{k}^{\prime}\right)+\bar{q}_{j}\left(q_{k}^{\prime}\right)=\left(\bar{q}_{i}+\bar{q}_{j}\right)(00 \ldots 0)_{p_{2}^{n}}$ and
(6) The number of " 0 ”s in $\bar{q}_{i}\left(q_{k}^{\prime}\right)+\bar{q}_{j}\left(q_{k}^{\prime}\right)$ is $\frac{p_{1}^{m}+1}{2}\left(p_{2}^{n}\right)$

The number of " 1 "s in $\bar{q}_{i}\left(q_{k}^{\prime}\right)+\bar{q}_{j}\left(q_{k}^{\prime}\right)$ is $\frac{p_{1}^{m}-1}{2}\left(p_{2}^{n}\right)$.

(7) The difference between the number of " $0 . s$ " and the number of " $1 . s$ " is $p_{2}^{n}$. Thus, $\bar{Q}_{1}\left(Q_{2}\right)$ are not orthogonal sets also $\bar{Q}_{1}\left(Q_{2}\right), Q_{1}\left(\bar{Q}_{2}\right)$, and $\bar{Q}_{1}\left(\bar{Q}_{2}\right)$ are not orthogonal sets.

e) If we redefine the addition on $A_{k}=Q_{1}\left(q_{k}^{\prime}\right)$ as following:

For $a_{i}, a_{j} \in A_{k}, a_{i} \oplus a_{j}=\left(q_{i}+q_{j}\right)\left(q_{k}^{\prime}\right)$ then the number of "1.s" in $a_{i} \oplus a_{j}$ is $\frac{p_{1}^{m} p_{2}^{n}+1}{2}$, the number of " $0 . \mathrm{s} "$ is $\frac{p_{1}^{m} p_{2}^{n}-1}{2}$, and the difference between the number of "1.s" and the number of " $0 . \mathrm{s}^{\mathrm{s}}$ " is one in this cause $\left(Q_{1}\left(Q_{2}\right), \oplus\right)$ and $\left.Q_{2}\left(Q_{1}\right), \oplus\right)$ are orthogonal sets.

f) Extending $Q_{1}, Q_{2}$ to $\tilde{Q}_{1}, \tilde{Q}_{2}$ respectively by adding "1" or " 0 "to the end (or starting) of each sequence in $Q_{1}, Q_{2}$, then $\tilde{Q}_{1}\left(\tilde{q}_{1}^{\prime}\right), \overline{\tilde{Q}}_{1}\left(\tilde{q}_{1}^{\prime}\right), \overline{\tilde{Q}}_{1}\left(\overline{\tilde{q}}_{1}\right)$ are not orthogonal sets.

\subsection{Compose Quotient Ring Sequences with Walsh Sequences and Increase}

\subsubsection{Compose Quotient Ring Sequences with Walsh Sequences}

Suppose $Q=\left\{q_{1}, q_{2}, \ldots, q_{u}\right\}$ is an orthogonal quotient ring sequences generated from binary representation of $Z_{p^{m}}$ and $W=\left\{w_{1}, w_{2}, \ldots, w_{2^{n}-1}\right\}$ is a Walsh sequences of order $2^{n}$ without zero sequences $w_{0}$ sequences, then in $Q(W)$.

Table 2. The numbers of "1.s" and "O.s" in each sequence of $Q$ and $W$.

\begin{tabular}{llll}
\hline $\boldsymbol{Q}$ & $\boldsymbol{W}$ & \\
\hline Number of "1.s" & Number of "0.s" & Number of "1.s" & Number of "0.s" \\
$\frac{p^{m}-1}{2}$ & $\frac{p^{m}+1}{2}$ & $2^{n-1}$ & $2^{n-1}$ \\
\hline
\end{tabular}

a) For $w_{k} \in W \quad$ we define the set $B_{k}=Q\left(w_{k}\right)=\left\{b_{i}=q_{i}\left(w_{k}\right), q_{i} \in Q\right\}$ then:

$\left(\frac{p^{m}-1}{2}\right)\left(2^{n-1}\right)+\left(\frac{p^{m}+1}{2}\right)\left(2^{n-1}\right)=2^{n-1} p^{m}$

(1) The number of " $1 . \mathrm{s}$ " in $b_{i}$ is $\left(\frac{p^{m}-1}{2}\right)\left(2^{n-1}\right)+\left(\frac{p^{m}+1}{2}\right)\left(2^{n-1}\right)=2^{n-1} p^{m}$

(2) The number of " $0 . \mathrm{s}$ " in $b_{i}$ is

(3) The difference between the number of " $1 . s$ " and the number of " $0 . \mathrm{s}$ " is zero

b) For $\quad b_{i}, b_{j} \in B_{k} \quad$ and $i \neq j$, $b_{i}+b_{j}=\left(q_{i}\right)\left(w_{k}\right)+\left(q_{j}\right)\left(w_{k}\right)=\left(q_{i}+q_{j}\right)(11 \ldots 1)_{2^{n}}$ and the 
difference between the number of "1.s" and the number of " $0 . \mathrm{s}$ " is $2^{n}$.

Thus $B_{k}$ is not orthogonal set and $(Q)(W)$ are not orthogonal sets.

c) if redefining on $Q(W)$ the operation $\oplus$ as following for $b_{i}, b_{j} \in B_{k}$ and $i \neq j, b_{i} \oplus b_{j}=\left(q_{i}+q_{j}\right)\left(w_{k}\right)$ in this cause.

The number of " $1 . \mathrm{s}$ " and the number of " $0 . \mathrm{s}$ " in $\tilde{b}_{i}$ is $2^{n-1} p^{m}$, the difference between them is zero.

The number of " $1 . \mathrm{s"}$ " in $b_{i} \oplus b_{j}$ is $2^{n-1} p^{m}$, the number of “0.s" in $b_{i} \oplus b_{j}$ is $2^{n-1} p^{m}$.

The difference between the number of " $1 . s$ "and the number of " $0 . \mathrm{s}$ " in $b_{i}$ and $b_{i} \oplus b_{j}$ is zero.

Thus $\left(B_{k}, \oplus\right)$ is an orthogonal set and $\left.Q(W), \oplus\right)$ are orthogonal sets.

\subsubsection{Compose Walsh Sequences with Quotient Ring Sequence}

Suppose $Q=\left\{q_{1}, q_{2}, \ldots, q_{u}\right\}$ is an orthogonal quotient ring sequences generated from binary representation of $Z_{p^{m}}$ and $W=\left\{w_{1}, w_{2}, \ldots, w_{2^{n}-1}\right\}$ is a Walsh sequences of order $2^{n}$ without zero sequences $w_{0}$ sequences, then in $W(Q)$

Table 3. The numbers of "1.s" and "O.s" in each sequence of $W$ and $Q$

\begin{tabular}{llll}
\hline $\boldsymbol{W}$ & $\boldsymbol{Q}$ \\
\hline $\begin{array}{l}\text { Number of "1.s" } \\
2^{n-1}\end{array}$ & Number of "0.s" & Number of "1.s" & Number of "0.s" \\
\hline
\end{tabular}

a) For $q_{k} \in Q \quad$ we define the set $\tilde{B}_{k}=W\left(q_{k}\right)=\left\{\tilde{b}_{i}=w_{i}\left(q_{k}\right), w_{i} \in W\right\}$ then

* The number of " $1 . \mathrm{s}$ " and the number of " $0 . \mathrm{s}$ " in $\tilde{b}_{i}$ is $2^{n-1} p^{m}$, the difference between them is zero.

$$
\text { For } \quad \tilde{b}_{i}, \tilde{b}_{j} \in \tilde{B}_{k} \quad \text { and } \quad i \neq j
$$

$\tilde{b}_{i}+\tilde{b}_{j}=\left(w_{i}\right)\left(q_{k}\right)+\left(w_{j}\right)\left(q_{k}\right)=\left(w_{i}+w_{j}\right)(11 . .1)_{p^{m}}$, the

number of " $1 . \mathrm{s}$ " and the number of " $0 . \mathrm{s}$ " in $\tilde{b}_{i}+\tilde{b}_{j}$ is $2^{n-1} p^{m}$, and the difference between the number of " $1 . s$ " and the number of " $0 . s$ " is zero.

Thus $\tilde{B}_{k}$ is an orthogonal set and $W(Q)$ are orthogonal sets.

b) If redefining on $W(Q)$ the operation $\oplus$ as following for $\tilde{b}_{i}, \tilde{b}_{j} \in \tilde{B}_{k}$ and $i \neq j, \tilde{b}_{i} \oplus \tilde{b}_{j}=\left(w_{i}+w_{j}\right)\left(q_{k}\right)$ in this case.

In $\tilde{b}_{i} \oplus \tilde{b}_{j}$, the number of " $1 . \mathrm{s}$ " is $2^{n-1} p^{m}$, the number of " $0 . \mathrm{s} "$ is $2^{n-1} p^{m}$, the difference between them is zero,
$\left(\tilde{B}_{k}, \oplus\right)$ is an orthogonal set, and $(W(Q), \oplus)$ are orthogonal sets.

\subsection{Compose Quotient Ring Sequences and M-Sequences and Inverse}

\subsubsection{Compose Quotient Ring Sequences and M-Sequences}

Suppose $Q=\left\{q_{1}, q_{2}, \ldots, q_{u}\right\}$ is an orthogonal quotient ring sequences generated from binary representation of $Z_{p^{m}}$ and $a_{1}$ is a non zero M-Sequence generated by the non homogeneous linear recurring sequence (1) of order $n$ with the prime characteristic polynomial.

$$
f(x)=x^{n}+\lambda_{n-1} x^{n-1}+\ldots+\lambda_{1} x+\lambda_{0}
$$

And the set $A=\left\{a_{i}, i=1,2, \ldots, 2^{n}-1\right\}$ of all cyclic shift of the sequence $a_{1}$ and the set $A$ form with the zero sequence an additive group, then in $Q(A)$.

Table 4. The numbers of " $1 . s$ " and "O.s" in each sequence of $Q$ and $A$.

\begin{tabular}{llll}
\hline $\boldsymbol{Q}$ & & $\mathbf{A}$ & \\
\hline Number of "1.s" & Number of " $0 . \mathrm{s} "$ & Number of " $1 . \mathrm{s} "$ & Number of " $0 . \mathrm{s}$ " \\
$\frac{p^{m}-1}{2}$ & $\frac{p^{m}+1}{2}$ & $2^{n-1}$ & $2^{n-1}-1$ \\
\hline
\end{tabular}

a) For $a_{k} \in A$ we define the set (2) The number of " $0 . \mathrm{s} "$ in $c_{i}$ is $C_{k}=Q\left(a_{k}\right)=\left\{c_{i}=q_{i}\left(a_{k}\right), q_{i} \in Q\right\}$ then.

(1) The number of "1.s" in $c_{i}$ is $\left(\frac{p^{m}-1}{2}\right)\left(2^{n-1}\right)+\left(\frac{p^{m}+1}{2}\right)\left(2^{n-1}-1\right)=\left(\frac{2^{n} p^{m}-p^{m}-1}{2}\right)$ $\left(\frac{p^{m}-1}{2}\right)\left(2^{n-1}-1\right)+\left(\frac{p^{m}+1}{2}\right)\left(2^{n-1}\right)=\left(\frac{2^{n} p^{m}-p^{m}+1}{2}\right)$

(3) The difference between the number of " $0 . s^{\text {" }}$ and the number of " $1 . \mathrm{s}$ " is one

(4) For $c_{i}, c_{j} \in C_{k}$ and $i \neq j, c_{i}+c_{j}=\left(q_{i}+q_{j}\right)(11 \ldots 1)_{2^{n}-1}$, 
the number of " $1 . \mathrm{s}$ " in $c_{i}+c_{j}$ is;

$\frac{\left(2^{n}-1\right)\left(p^{m}-1\right)}{2}$, the number of " $0 . \mathrm{s}$ " in $c_{i}+c_{j}$ is $\frac{\left(2^{n}-1\right)\left(p^{m}+1\right)}{2}$, and the difference between the number of " $0 . \mathrm{s}$ " and the number of " $1 . \mathrm{s}$ " is $2^{n}-1$.

Thus $C_{k}$ is not orthogonal set and $Q(A)$ are not orthogonal sets.

b) Redefining on $Q(A)$ the operation $\oplus$ as following for $c_{i}, c_{j} \in C_{k}$ and $i \neq j, c_{i} \oplus c_{j}=\left(q_{i}+q_{j}\right)\left(a_{k}\right)$, in $c_{i} \oplus c_{j}$ the number of " $1 . \mathrm{s}$ " is $\frac{2^{n} p^{m}-p^{m}-1}{2}$, the number of " $0 . \mathrm{s}$ " is $\frac{2^{n} p^{m}-p^{m}+1}{2}$, and the difference between the number of " $0 . s^{\text {" }}$ and the number of " $1 . \mathrm{s}$ " is one.

Thus $\left(C_{k}, \oplus\right)$ is an orthogonal set and $(Q(A), \oplus)$ are orthogonal sets.

\subsubsection{Compose M-Sequences and Quotient Ring Sequences and Finding $A(Q)$}

Table 5. The numbers of "1.s" and "O.s" in each sequence of $A$ and $Q$.

\begin{tabular}{llll}
\hline $\mathbf{A}$ & $\boldsymbol{Q}$ & \\
\hline Number of "1.s" & Number of "0.s" & Number of "1.s" & Number of "0.s" \\
$2^{n-1}$ & $2^{n-1}-1$ & $\left(p^{m}-1\right) / 2$ & $\left(p^{m}+1\right) / 2$ \\
\hline
\end{tabular}

a) For $q_{k} \in Q \quad$ we define the set $\tilde{C}_{k}=A\left(q_{k}\right)=\left\{\tilde{c}_{i}=a_{i}\left(q_{k}\right), a_{i} \in A\right\}$ then.

(1) The number of " $1 . \mathrm{s}$ " in $c_{i}$ is $\left(\frac{2^{n} p^{m}-p^{m}-1}{2}\right)$, the number of " $0 . \mathrm{s}$ " in $c_{i}$ is $\left(\frac{2^{n} p^{m}-p^{m}+1}{2}\right)$.

(2) The difference between the number of " $0 . s$ " and the number of " $1 . s$ " is one

b) For $\tilde{c}_{i}, \tilde{c}_{j} \in \tilde{C}_{k}$ and $i \neq j, \tilde{c}_{i}+\tilde{c}_{j}=\left(a_{i}+a_{j}\right)(11 \ldots 1)_{p^{m}}$, in $\tilde{c}_{i}+\tilde{c}_{j}$ the number of " $1 . \mathrm{s}$ " is

$2^{n-1} p^{m}$, the number of " $0 . \mathrm{s}$ " is $\left(2^{n-1}-1\right) p^{m}$, and the difference between the number of " $1 . s$ " and the number of "0.s" is $p^{m}$.
Thus $\tilde{C}_{k}$ is not orthogonal set and $A(Q)$ are not orthogonal sets.

c) Redefining on $A(Q)$ the operation $\oplus$ as following for $q_{k} \in Q$ and $i \neq j, \tilde{c}_{i} \oplus \tilde{c}_{j}=\left(a_{i}+a_{j}\right)\left(q_{k}\right)$, in $\tilde{c}_{i} \oplus \tilde{c}_{j}$ the number of " $1 . \mathrm{s}$ " is $\frac{2^{n} p^{m}-p^{m}-1}{2}$, the number of " $0 . \mathrm{s}$ " is $\frac{2^{n} p^{m}-p^{m}+1}{2}$, and the difference between the number of "0.s"and the number of " $1 . s$ " is one.

Thus $\left(\tilde{C}_{k}, \oplus\right)$ is an orthogonal set and $\left.A(Q), \oplus\right)$ are orthogonal sets.

Example 1. For $p=5$, Table 1. Contains the multiplication on $Z_{5}$ and their binary representation.

Table 6. .Multiplication on $Z_{5}$ and their binary representation.

\begin{tabular}{|c|c|c|c|c|c|c|c|c|c|c|c|c|c|c|}
\hline & $*$ & 0 & 1 & 2 & 3 & 4 & \multirow{6}{*}{$\Rightarrow$} & & $*$ & 0 & 1 & 2 & 3 & 4 \\
\hline R0 & 0 & 0 & 0 & 0 & 0 & 0 & & $r_{0}$ & 0 & 0 & 0 & 0 & 0 & 0 \\
\hline R1 & 1 & 0 & 1 & 2 & 3 & 4 & & $r_{1}$ & 1 & 0 & 1 & 0 & 1 & 0 \\
\hline R2 & 2 & 0 & 2 & 4 & 1 & 3 & & $r_{2}$ & 2 & 0 & 0 & 0 & 1 & 1 \\
\hline R3 & 3 & 0 & 3 & 1 & 4 & 2 & & $r_{3}$ & 3 & 0 & 1 & 1 & 0 & 0 \\
\hline R4 & 4 & 0 & 4 & 3 & 2 & 1 & & $r_{4}$ & 4 & 0 & 0 & 1 & 0 & 1 \\
\hline
\end{tabular}

Each of $r_{1}$ and $r_{2}$ contains $3=\frac{5+1}{2}$ of " $0 . \mathrm{s}$ " and $2=\frac{5-1}{2}$ of " $1 . s$ " and $r_{1}+r_{2}$ contains also 3 of " $0 . s$ " and 2 of "1.s", but $r_{1}+r_{4}=r_{2}+r_{3}=\left[\begin{array}{lllll}0 & 1 & 1 & 1 & 1\end{array}\right]$, where " + " is the ordinary addition and performed by $\bmod 2$, span $\left\{r_{1}, r_{2}\right\}$, without $r_{0}=q_{0}$, is $Q_{1}=\left\{q_{1}=r_{1}, q_{2}=r_{2}, q_{3}=r_{1}+r_{2}\right\}$ is a biggest orthogonal set, where $q_{1}=(01010), q_{2}=(00011)$, $q_{3}=(01001)$, and $\operatorname{Span}\left\{r_{1}, r_{2}\right\}=\left\{q_{0}, q_{1}, q_{2}, q_{3}\right\}$ is a subgroup in the binary vector space of order $2^{5}$ for addition and $q_{2}=(00011), q_{3}=(01001)$, and $\operatorname{Span}\left\{r_{1}, r_{2}\right\}=\left\{q_{0}\right.$, $\left.q_{1}, q_{2}, q_{3}\right\}$ is a subgroup in the binary vector space of order $2^{5}$ for addition and. 
Table 7. Span $\left\{r_{1}, r_{2}\right\}$ without $q_{0}=r_{0}$.

\begin{tabular}{llllll}
\hline$q_{1}$ & 0 & 1 & 0 & 1 \\
$q_{2}$ & 0 & 0 & 0 & 1 \\
$q_{3}$ & 0 & 1 & 0 & 0 & 1 \\
\hline
\end{tabular}

Example 2. For $p=3$, Table 2 showing binary representation of $Z_{3^{2}}$

Table 8. Binary Representation of $Z_{3^{2}}$.

\begin{tabular}{|c|c|c|c|c|c|c|c|c|c|c|}
\hline & $*$ & $\mathbf{0}$ & 1 & 2 & 3 & 4 & 5 & 6 & 7 & 8 \\
\hline$r_{0}^{\prime}$ & 0 & 0 & 0 & 0 & 0 & 0 & 0 & 0 & 0 & 0 \\
\hline$\eta^{\prime}$ & 1 & 0 & 1 & 0 & 1 & 0 & 1 & 0 & 1 & 0 \\
\hline$r_{2}$ & 2 & 0 & 0 & 0 & 0 & 0 & 1 & 1 & 1 & 1 \\
\hline$r_{3}$ & 3 & 0 & 1 & 0 & 0 & 1 & 0 & 0 & 1 & 0 \\
\hline$r_{4}^{\prime}$ & 4 & 0 & 0 & 0 & 1 & 1 & 0 & 0 & 1 & 1 \\
\hline$r_{5}^{\prime}$ & 5 & 0 & 1 & 1 & 0 & 0 & 1 & 1 & 0 & 0 \\
\hline$r_{6}^{\prime}$ & 6 & 0 & 0 & 1 & 0 & 0 & 1 & 0 & 0 & 1 \\
\hline$r_{7}$ & 7 & 0 & 1 & 1 & 1 & 1 & 0 & 0 & 0 & 0 \\
\hline$r_{8}^{\prime}$ & 8 & 0 & 0 & 1 & 0 & 1 & 0 & 1 & 0 & 1 \\
\hline
\end{tabular}

We can see that $\operatorname{Span}\left\{r_{1}^{\prime}, r_{2}^{\prime}, r_{4}^{\prime}\right\}=\left\{r_{1}^{\prime}, r_{2}^{\prime}, r_{4}^{\prime}, r_{1}^{\prime}+r_{2}^{\prime}, r_{1}^{\prime}+r_{4}^{\prime}, r_{2}^{\prime}+r_{4}^{\prime}\right\}$ is a maximum closed orthogonal set contained in $F_{2^{9}}$

Thus $Q_{2}=\left\{q_{1}^{\prime}, q_{2}^{\prime}, q_{3}^{\prime}, q_{4}^{\prime}, q_{5}^{\prime}, q_{6}^{\prime}\right\} \quad$ where $\quad q_{1}^{\prime}=r_{1}^{\prime}=(010101010) \quad, \quad q_{2}^{\prime}=r_{2}^{\prime}=(000001111) \quad, \quad q_{3}^{\prime}=r_{3}^{\prime}=(000110011)$, $q_{4}^{\prime}=(010100101), q_{5}^{\prime}=(010011001), q_{6}^{\prime}=(000111100)$.

a) Finding $Q_{1}\left(q_{1}^{\prime}\right)$, where $q_{1}^{\prime}=(010101010), \overline{q_{1}^{\prime}}=(101010101)$.

Table 9. Compose $Q_{1}$ with $q_{1}^{\prime}$ from $Q_{2}$.

\begin{tabular}{lccccc}
\hline$q_{1}\left(q_{1}^{\prime}\right)$ & 101010101 & 010101010 & 101010101 & 010101010 & 101010101 \\
$q_{2}\left(q_{1}^{\prime}\right)$ & 101010101 & 101010101 & 101010101 & 010101010 & 010101010 \\
$q_{3}\left(q_{1}^{\prime}\right)$ & 101010101 & 010101010 & 101010101 & 101010101 & 010101010 \\
$a_{1}+a_{2}=q_{1}\left(q_{1}^{\prime}\right)+q_{2}\left(q_{1}^{\prime}\right\}$ & 000000000 & 111111111 & 000000000 & 000000000 \\
\hline
\end{tabular}

Thus $p_{1}=5, m_{1}=1, p_{2}=3, m_{2}=2$ and.

Each row contains $\frac{p_{1}^{m} p_{2}^{n}+1}{2}=\frac{5\left(3^{2}\right)+1}{2}=23$ of " $1 . \mathrm{s}$ ", and $\frac{p_{1}^{m} p_{2}^{n}-1}{2}=\frac{5\left(3^{2}\right)-1}{2}=22$ of " $0 . \mathrm{s}$ ".

The difference between the number of " $1 . s$ " and the number of " $0 . \mathrm{s}$ is one but $q_{1}\left(q_{1}^{\prime}\right)+q_{2}\left(q_{1}^{\prime}\right)$ contains 18 of " $0 . s$ ", 27 of " $0 . s$ " and the difference between the number of "0.s" and the number of " $1 . \mathrm{s"}$ " is $3^{2}=9$ and $A_{k}=Q_{1}\left(q_{k}^{\prime}\right)$ not orthogonal set or $Q_{1}\left(Q_{2}\right)$ are not orthogonal sets.

b) If we redefine the addition on $A_{k}=Q_{1}\left(q_{k}^{\prime}\right)$ as following, For $a_{i}, a_{j} \in A_{k}, a_{i} \oplus a_{j}=\left(q_{i}+q_{j}\right)\left(q_{k}\right)$ then, the number of "1.s" in $a_{i} \oplus a_{j}$ is $\frac{p_{1}^{m} p_{2}^{n}+1}{2}$, the number of " $0 . \mathrm{s}$ " is $\frac{p_{1}^{m} p_{2}^{n}-1}{2}$ and the difference between the number of " $1 . s "$ and the number of " 0 "s is one.

Table 10. Compose $q_{1}$ and $q_{2}$ from $Q_{1}$.

\begin{tabular}{lllll}
\hline$a_{1} \oplus a_{2}=\left(q_{1}+q_{2}\right)\left(q_{1}^{\prime}\right)=q_{3}\left(q_{1}^{\prime}\right)$ & 101010101 & 010101010 & 101010101 & 101010101 \\
\hline
\end{tabular}

$a_{1} \oplus a_{2}$ Contains 23 of " $1 . s ", 22$ of " $0 . \mathrm{s}$ " thus, $A_{k}=Q_{1}\left(q_{k}^{\prime}\right)$ is an orthogonal set and $Q_{1}\left(Q_{2}\right)$ with the 
operation $\oplus$ in this case are orthogonal sets.

c) Also $\bar{q}_{i}\left(q_{k}\right)+\bar{q}_{j}\left(q_{k}\right)$ is not orthogonal set, For example,

calculate $\bar{q}_{1}\left(q_{1}\right)+\bar{q}_{2}\left(q_{1}\right)$ we have.

Table 11. Complements of $q_{1}$ and $q_{2}$ from $Q_{1}$.

\begin{tabular}{llllll}
\hline $\bar{q}_{1}$ & 1 & 0 & 1 & 0 & 1 \\
$\bar{q}_{2}$ & 1 & 1 & 1 & 0 & 0 \\
\hline & & Table 12. $\operatorname{Span}^{*}\left\{\bar{q}_{1}\left(q_{1}^{\prime}\right), \bar{q}_{2}\left(q_{1}^{\prime}\right)\right\}$. & & \\
\hline $\bar{q}_{1}\left(q_{1}^{\prime}\right)$ & 010101010 & 101010101 & 010101010 & 101010101 & 011010101 \\
$\bar{q}_{2}\left(q_{1}^{\prime}\right)$ & 010101010 & 010101010 & 010101010 & 101010101 & 101010101 \\
$\bar{q}_{1}\left(q_{1}^{\prime}\right)+\bar{q}_{2}\left(q_{1}^{\prime}\right)$ & 000000000 & 111111111 & 000000000 & 000000000 & 111111111 \\
\hline
\end{tabular}

Thus, $\bar{q}_{1}\left(q_{1}^{\prime}\right)+\bar{q}_{2}\left(q_{1}^{\prime}\right)=\left(\bar{q}_{1}+\bar{q}_{2}\right)(111111111)$ and $\bar{Q}_{1}\left(q_{1}\right)$ is not orthogonal set or $\bar{Q}_{1}\left(Q_{2}\right)$ are not orthogonal sets.

d) Extending $Q_{1}, Q_{2}$ to $\tilde{Q}_{1}, \tilde{Q}_{2}$ respectively by adding "1" or " 0 "to the end (or starting) of each sequence in $Q_{1}, Q_{2}$, then $\tilde{Q}_{1}\left(q_{1}^{\prime}\right), \tilde{Q}_{1}\left(\tilde{q}_{1}^{\prime}\right), \overline{\tilde{Q}}_{1}\left(\tilde{q}_{1}^{\prime}\right), \overline{\tilde{Q}}_{1}\left(\overline{\tilde{q}}_{1}\right)$ are not orthogonal sets, for example:

Table 13. Extending $q_{1}$ and $q_{2}$ from $Q_{1}$.

\begin{tabular}{lllllll}
\hline$\tilde{q}_{1}$ & 0 & 1 & 0 & 1 & 0 & 0 \\
$\tilde{q}_{2}$ & 0 & 0 & 0 & 1 & 1 & 0 \\
\hline
\end{tabular}

Table 14. $\operatorname{Span}^{*}\left\{\tilde{q}_{1}\left(q_{1}^{\prime}\right), \tilde{q}_{2}\left(q_{1}^{\prime}\right)\right\}$.

\begin{tabular}{lllllll}
\hline$\tilde{q}_{1}\left(q_{1}{ }^{\prime}\right)$ & 101010101 & 010101010 & 101010101 & 010101010 & 101010101 & 101010101 \\
$\tilde{q}_{2}\left(q_{1}{ }^{\prime}\right)$ & 101010101 & 101010101 & 101010101 & 010101010 & 010101010 & 101010101 \\
$\tilde{q}_{1}\left(q_{1}{ }^{\prime}\right)+\tilde{q}_{2}\left(q_{1}{ }^{\prime}\right)$ & 000000000 & 111111111 & 0000000000 & 000000000 & 111111111 & 000000000 \\
\hline
\end{tabular}

Thus, $\tilde{q}_{1}\left(q_{1}^{\prime}\right)+\tilde{q}_{2}\left(q_{1}^{\prime}\right)=\left(\tilde{q}_{1}+\tilde{q}_{2}\right)(000000000)$ and $\tilde{Q}_{1}\left(q_{1}\right)$ is not orthogonal set or $\tilde{Q}_{1}\left(Q_{2}\right)$ are not orthogonal sets.

Example 3. The following table showing Walsh sequences of order $8=2^{3}$ without null sequence.

\section{Table 15. Walsh Sequences of order $8=2^{3}$}

$$
\begin{aligned}
& w_{1}=000011111 \\
& w_{1}=000001111 \\
& w_{1}=000001111 \\
& w_{4}=011100110 \\
& w_{5}=0111011001 \\
& w_{6}=0110111010 \\
& w_{7}=01101101101
\end{aligned}
$$

Suppose $Q$ is as in example1, $W$ is a set of Walsh Sequences of order $2^{n}$,

a) Compose $Q$ with $\mathrm{W}$ or $Q(W)$ and $B_{k}=Q\left(w_{k}\right)=\left\{b_{i}=q_{i}\left(w_{k}\right), q_{i} \in Q\right\} \quad, \quad$ (for example $Q\left(W_{2^{3}}\right)$ and $\left.B_{1}\right)$ then.

The number of " $1 . \mathrm{s}$ " of each $b_{i}$ is $2^{n-1} p^{m}$, (in the example $\left.=2^{2}(5)=20\right)$.

\begin{tabular}{|c|c|c|c|c|c|}
\hline$q_{1}\left(w_{1}\right)$ & 10101010 & 01010101 & 10101010 & 01010101 & 10101010 \\
\hline$q_{2}\left(w_{1}\right)$ & 10101010 & 10101010 & 10101010 & 01010101 & 01010101 \\
\hline$q_{3}\left(w_{1}\right)$ & 10101010 & 01010101 & 10101010 & 10101010 & 01010101 \\
\hline$q_{1}\left(w_{1}\right)+q_{2}\left(w_{1}\right)$ & 00000000 & 11111111 & 00000000 & 00000000 & 11111111 \\
\hline
\end{tabular}

The number of " $0 . \mathrm{s}$ " of each $b_{i}$ is $2^{n-1} p^{m}$, (in the example $=2^{2}(5)=20$ ).

The difference between the number of " $1 . s$ " and the number of " $0 . \mathrm{s}$ " is zero. For $w_{1}=(01010101), w_{2}=$ (10101010).

Table 16. Compose $Q_{1}$ with $w_{1}$ from $W$

Thus, $q_{1}\left(w_{1}\right)_{+} q_{2}\left(w_{1}\right)$ contains 16 of " $1 . \mathrm{s}$ " and 24 of " $0 . \mathrm{s}$ " and the difference between the number of " $1 . \mathrm{s}$ " and the number of " $0 . \mathrm{s}$ " is $2^{n}=2^{3}=8$, and $Q\left(W_{2^{3}}\right)$ or $Q(W)$ are not orthogonal sets. 
Redefining on $Q(W)$ the operation $\oplus$ as following for (in the example $\left.b_{1} \oplus b_{2}=\left(q_{1}+q_{2}\right)\left(w_{1}\right)=q_{3}\left(w_{1}\right)\right)$ and. $b_{i}, b_{j} \in B_{k}$ and $i \neq j$ as $b_{i} \oplus b_{j}=\left(q_{i}+q_{j}\right)\left(w_{k}\right)$ in this case

Table 17. Compose $q_{1}+q_{2}=q_{3}$ with $w_{1}$ from $W$.

\begin{tabular}{|c|c|c|c|c|c|}
\hline$q_{1} \oplus q_{2}=q_{3}\left(w_{1}\right)$ & 10101010 & 01010101 & 10101010 & 10101010 & 01010101 \\
\hline
\end{tabular}

The number of " 1 "s in $b_{i} \oplus b_{j}$ is $2^{n-1} p^{m}$, (In the example $\left.=20\right)$

The number of " 0 "s in $b_{i} \oplus b_{j}$ is. $2^{n-1} p^{m},($ In the example $=20)$

The difference between the number of "1.s" and the number of " $0 . \mathrm{s}$ " in $b_{i}$ and $b_{i} \oplus b_{j}$ is zero.

Thus $B_{k}$ is an orthogonal set and $(Q)(W)$ are orthogonal sets.

Example 4. Compose Walsh sequences with quotient ring sequences.

a) Compose $\mathrm{W}$ with $Q$ or $W(Q)$ and $\tilde{B}_{k}=W\left(q_{k}\right)=\left\{\tilde{b}_{i}=w_{i}\left(q_{i}\right), w_{i} \in W\right\}$ (for example $W_{2^{3}}(Q)$ and $\tilde{B}_{1}$ ) then.

The number of " $1 . \mathrm{s}$ " of each $\tilde{b}_{i}$ is $2^{n-1} p^{m}$, (in the example $=2^{2}(5)=20$ ).

The number of " $0 . \mathrm{s}$ " of each $\tilde{b}_{i}$ is $2^{n-1} p^{m}$, (in the example $=2^{2}(5)=20$ ).

The difference between the number of "1.s" and the number of " $0 . \mathrm{s}$ " is zero, $q_{1}=(01010), \bar{q}_{1}=(10101)$ and.

Table 18. Compose $W$ with $q_{1}$ from $Q_{1}$.

\begin{tabular}{lllllllll}
\hline$w_{1}\left(q_{1}\right)$ & 10101 & 10101 & 10101 & 10101 & 01010 & 01010 & 01010 & 01010 \\
$w_{2}\left(q_{1}\right)$ & 10101 & 10101 & 01010 & 01010 & 01010 & 01010 & 10101 & 10101 \\
$w_{3}\left(q_{1}\right)$ & 10101 & 10101 & 01010 & 01010 & 10101 & 10101 & 01010 & 01010 \\
$w_{4}\left(q_{1}\right)$ & 10101 & 01010 & 01010 & 10101 & 10101 & 01010 & 01010 & 10101 \\
$w_{5}\left(q_{1}\right)$ & 10101 & 01010 & 01010 & 10101 & 01010 & 10101 & 10101 & 01010 \\
$w_{6}\left(q_{1}\right)$ & 10101 & 01010 & 10101 & 01010 & 01010 & 10101 & 01010 & 10101 \\
$w_{7}\left(q_{1}\right)$ & 10101 & 01010 & 10101 & 01010 & 10101 & 01010 & 10101 & 01010 \\
$w_{1}\left(q_{1}\right)+w_{2}\left(q_{1}\right)=w_{3}\left(q_{1}\right)$ & 00000 & 00000 & 11111 & 11111 & 00000 & 00000 & 11111 & 11111 \\
\hline
\end{tabular}

For $i \neq j$ in $\tilde{b}_{i}+\tilde{b}_{j}$, the number of " $1 . \mathrm{s}$ " and the number of " $0 . \mathrm{s}$ " is $2^{n-1} p^{m}=20$, and the difference between the number of " $1 . \mathrm{s}$ " and the number of " $0 . \mathrm{s}$ " is zero.

Thus $\left(\tilde{B}_{k},+\right)$ is an orthogonal set and $(W(Q),+)$ are orthogonal sets. b) Redefining on $W(Q)$ the operation ${ }^{\oplus}$ as following for $\tilde{b}_{i}, \tilde{b}_{j} \in \tilde{B}_{k}$ and $i \neq j$ as $\tilde{b}_{i} \oplus \tilde{b}_{j}=\left(w_{i}+w_{j}\right)\left(q_{k}\right)$ in this case (in the example $\tilde{b}_{1} \oplus \tilde{b}_{2}=\left(w_{1}+w_{2}\right)\left(q_{1}\right)=w_{3}\left(q_{1}\right)$ ).

Table 19. Compose $w_{1}+w_{2}=w_{3}$ from $W$ with $q_{1}$ from $Q_{1}$

\begin{tabular}{llllllll}
\hline$\tilde{b}_{1} \oplus \tilde{b}_{2}=w_{3}\left(q_{1}\right)$ & 10101 & 10101 & 01010 & 01010 & 10101 & 10101 & 01010 \\
\hline
\end{tabular}

(4) The number of “ $1 . \mathrm{s}$ " in $\tilde{b}_{i} \oplus \tilde{b}_{j}$ is $2^{n-1} p^{m}$, (In example $=20$ )

(5) The number of " $0 . \mathrm{s}$ " in $\tilde{b}_{i} \oplus \tilde{b}_{j}$ is $2^{n-1} p^{m}$, (In example $=20$ ).

(6) The difference between the number of " $1 . \mathrm{s}$ " and the number of " 0. s" $^{\text {" in }} b_{i}$ and $\tilde{b}_{i} \oplus \tilde{b}_{j}$ is zero.

Thus $\tilde{B}_{k}$ is an orthogonal set and $W(Q)$ are orthogonal sets.

Example 5. Given $Q=\left\{q_{1}, q_{2}, q_{3}\right\}$ orthogonal quotient ring sequences generated from binary representation of $Z_{5}$ (as in the example1., $p=5$ and $m=1$ ) and $a_{1}$ is a non zero MSequence generated by the non homogeneous linear recurring sequence.

$$
z_{n+2}=z_{n+1}+z_{n} \text { or } z_{n+2}=z_{n+1}+z_{n}
$$

With the characteristic equation $x^{2}+x+1=0$ and the characteristic polynomial $f(x)=x^{2}+x+1$ the set $A=\left\{a_{1}, a_{2}, a_{3}\right\}$ where $a_{1}=(101), a_{2}=(110), a_{3}=(011)$, and the first two digits in each sequence are the initial position of the feedback register, and the set $A$ is an orthogonal set.

Table 20. $A=$ M-Sequences of order 3.

\begin{tabular}{llll}
\hline$a_{1}$ & 1 & 0 & 1 \\
$a_{2}$ & 1 & 1 & 0 \\
$a_{3}$ & 0 & 1 & 1 \\
\hline
\end{tabular}


Compos $Q$ and $A$ or finding $Q(A) \quad a_{1}=(101), \overline{a_{1}}=(010)$

Table 21. Elements $Q_{1}$.

\begin{tabular}{llllll}
\hline$q_{1}$ & 0 & 1 & 0 & 1 & 0 \\
$q_{2}$ & 0 & 0 & 0 & 1 & 1 \\
$q_{3}$ & 0 & 1 & 0 & 0 & 1 \\
\hline
\end{tabular}

Table 22. Compose $Q_{1}$ with $a_{1}$ from $A$.

\begin{tabular}{llllll}
\hline$q_{1}\left(a_{1}\right)$ & 010 & 101 & 010 & 101 & 010 \\
$q_{2}\left(a_{1}\right)$ & 010 & 010 & 010 & 101 & 101 \\
$q_{3}\left(a_{1}\right)$ & 010 & 101 & 010 & 010 & 101 \\
$q_{1}\left(a_{1}\right)+q_{2}\left(a_{1}\right)=q_{3}\left(a_{1}\right)$ & 000 & 111 & 000 & 000 & 111 \\
\hline
\end{tabular}

a) For $a_{k} \in A$ we define the set $\left.C_{k}=Q\left(a_{k}\right)=c_{i}=q_{i}\left(a_{k}\right), q_{i} \in Q\right\}$ then.

(1) The number of " $1 . \mathrm{s}$ " in $c_{i}$ is $\left(\frac{2^{n} p^{m}-p^{m}-1}{2}\right)=\frac{4(5)-5-1}{2}=7$

(2) The number of " $0 . \mathrm{s}$ " in $c_{i}$ is $\left(\frac{2^{n} p^{m}-p^{m}-1}{2}\right)=\frac{4(5)-5+1}{2}=8$

(3) The difference between the number of " $0 . \mathrm{s}^{\text {" }}$ and the number of " $1 . \mathrm{s}$ " is one.

b) For $c_{i}, c_{j} \in C_{k}$ and $i \neq j$ the $c_{i}+c_{j}=\left(q_{i}+q_{j}\right)(111)$ and the number of " $1 . \mathrm{s}$ " in $c_{i}+c_{j}$ is

$\frac{\left(2^{n}-1\right)\left(p^{m}-1\right)}{2}=6$ the number of " $0 . \mathrm{s}$ " in $c_{i}+c_{j}$ is $\frac{\left(2^{n}-1\right)\left(p^{m}+1\right)}{2}=9$ and the difference

between the number of " $0 . \mathrm{s}$ " and the number of " $1 . \mathrm{s}$ " is $2^{n}-1=3$.

Thus $C_{k}$ is not orthogonal set and $Q(A)$ are not orthogonal sets.

c) Redefining on $Q(A)$ the operation $\oplus$ as following for $c_{i}, c_{j} \in C_{k}$ and $i \neq j$ as $c_{i} \oplus c_{j}=\left(q_{i}+q_{j}\right)\left(a_{k}\right)$ in this case (in the example $\left.c_{1} \oplus c_{2}=\left(q_{1}+q_{2}\right)\left(a_{1}\right)=q_{3}\left(a_{1}\right)\right)$ and.

Table 23. Compose $q_{1}+q_{2}=q_{3}$ from $Q_{1}$ with $a_{1}$ from $A$.

\begin{tabular}{llllll}
\hline$c_{1} \oplus c_{2}=q_{3}\left(a_{1}\right)$ & 010 & 101 & 010 & 101 & 010 \\
\hline
\end{tabular}

The number of " $1 . \mathrm{s}$ " in $c_{i} \oplus c_{j}$ is $\frac{2^{n} p^{m}-p^{m}-1}{2}$, (In example $=7$ )

The number of " $0 . \mathrm{s}$ " in $c_{i} \oplus c_{j}$ is $\frac{2^{n} p^{m}-p^{m}+1}{2}$, (In example $=8$ )

The difference between the number of " $0 . \mathrm{s}$ " and the number of " $1 . \mathrm{s}$ " in $c_{i} \oplus c_{j}$ is one.

Thus $\left(C_{k}, \oplus\right)$ is an orthogonal set and $(Q(A), \oplus)$ are orthogonal sets.

d) For $\left.\tilde{C}_{k}=A\left(q_{k}\right)=\tilde{c}_{i}=a_{i}\left(q_{k}\right), a_{i} \in A\right\}$

(7) The number of "1.s" in $\tilde{c}_{i}$ is $\left(\frac{2^{n} p^{m}-p^{m}-1}{2}\right)=\frac{4(5)-5-1}{2}=7$

(8) The number of " $0 . \mathrm{s}$ " in $\tilde{c}_{i}$ is $\left(\frac{2^{n} p^{m}-p^{m}-1}{2}\right)=\frac{4(5)-5+1}{2}=8$

(9) The difference between the number of " $0 . s$ " and the number of " $1 . s$ " is one.

e) For $\tilde{c}_{i}, \tilde{c}_{j} \in \tilde{C}_{k}$ and $i \neq j$ the $\tilde{c}_{i}+\tilde{c}_{j}=\left(a_{i}+a_{j}\right)(11 \ldots 1)_{p^{m}}$ in $\tilde{c}_{i}+\tilde{c}_{j}$ the number of " $1 . \mathrm{s"}$ " is

$\frac{\left(2^{n}-1\right)\left(p^{m}-1\right)}{2}=6$ the number of " $0 . \mathrm{s}$ " is $\frac{\left(2^{n}-1\right)\left(p^{m}+1\right)}{2}=9$ and the difference between the number of " $0 . \mathrm{s}$ " and the number of " $1 . \mathrm{s}$ " is $p^{m}=3^{1}=3$. 
Thus $\tilde{C}_{k}$ is not orthogonal set and $A(Q)$ are not orthogonal sets.

f) Redefining on $A(Q)$ the operation $\oplus$ as following for $\tilde{c}_{i}, \tilde{c}_{j} \in \tilde{C}_{k}$ and $i \neq j$ as $\tilde{c}_{i} \oplus \tilde{c}_{j}=\left(a_{i}+a_{j}\right)\left(q_{k}\right)$ in this case (in the example $\tilde{c}_{1} \oplus \tilde{c}_{2}=\left(a_{1}+a_{2}\right)\left(q_{1}\right)=a_{3}\left(q_{1}\right)$ )

Table 24. Compose $a_{1}+a_{2}=a_{3}$ from $A$ with $q_{1}$ from $Q_{1}$.

\begin{tabular}{lcll}
\hline$\tilde{c}_{1} \oplus \tilde{c}_{2}=a_{3}\left(q_{1}\right)$ & 10101 & 01010 & 01010 \\
\hline The number of " 1 ”s in $\tilde{c}_{i} \oplus \tilde{c}_{j}$ is $\frac{2^{n} p^{m}-p^{m}-1}{2}$, (In
\end{tabular}
example $=7$ )

The number of " 0 "s in $\tilde{c}_{i} \oplus \tilde{c}_{j}$ is $\frac{2^{n} p^{m}-p^{m}+1}{2}$, (In example $=8$ )

The difference between the number of " $0 . s$ " and the number of " $1 . \mathrm{s}$ " in $\tilde{c}_{i} \oplus \tilde{c}_{j}$ is one.

Thus $\left(\tilde{C}_{k}, \oplus\right)$ is an orthogonal set and $(A(Q), \oplus)$ are orthogonal sets.

\section{Conclusion}

Suppose $Q$ is an orthogonal quotient ring obtained from binary representation of $Z_{p^{m}}, \mathrm{~W}$ is a Walsh sequences of order $2^{n}$, and $A$ is a M-Sequences, + is the ordinary addition $\bmod 2$, and $\oplus$ is a special addition $\bmod 2$ then

\subsection{For Compose Quotient Ring Sequences with Other Quotient Ring Sequences}

The $\left(Q_{1}\left(Q_{2}\right),+\right)$ and $\left(Q_{2}\left(Q_{1}\right),+\right)$ are not orthogonal sets, $\left(Q_{1}\left(Q_{2}\right), \oplus\right)$ and $\left(Q_{2}\left(Q_{1}\right), \oplus\right)$ are orthogonal sets with the length $N=p_{1}^{m} p_{2}^{n}$, minimum distance $d=\frac{p_{1}^{m} p_{2}^{n}+1}{2}$, not linear, not cyclic, and dimension $k \geq m, k \geq n$ respectively.

For compose quotient ring sequences with other Walsh's Sequences or in inverse

(a) The $(Q(W),+)$ are not orthogonal sets, $(Q(W), \oplus)$ are orthogonal sets with the length $N=2^{n} p^{m}$, minimum distance $d=2^{n-1} p^{m}$, not linear, not cyclic, and dimension $k \geq m$

(b) The $(W(Q),+)$ and $(W(Q), \oplus)$ are orthogonal sets with the length $N=2^{n} p^{m}$, minimum distance $d=2^{n-1} p^{m}$, not linear, not cyclic, and dimension $k \geq n$.

\subsection{For Compose Quotient Ring Sequences with Other M- Sequences or in Inverse}

(c) The $(Q(A),+)$ are not orthogonal sets, $(Q(A), \oplus)$ are orthogonal sets with the length $N=\left(2^{n}-1\right) p^{m}$, minimum distance

$$
d=\frac{2^{n} p^{m}-p^{m}-1}{2}, \text { not linear, not cyclic, and dimension }
$$
$k \geq m$.

(d) The $(A(Q),+)$ are not orthogonal sets, $(A(Q), \oplus)$ are orthogonal sets with the length $N=\left(2^{n}-1\right) p^{m}$, minimum distance $d=\frac{2^{n} p^{m}-p^{m}-1}{2}$, not linear, not cyclic, and dimension $k \geq n$.

Thus, sequence generated showed increased secrecy and increased possibility of correcting error in communication channel because it exhibited bigger length and the bigger minimum distance.

\section{Limitation}

This method of compose sequences is useful for only binary sequences and the addition on the sequences computed by "mod 2" also used Microsoft Word 2010 and the Microsoft equation 3.0 for written the math equations.

The method for reading a page which has a block will be according to the following direction as in figure1.

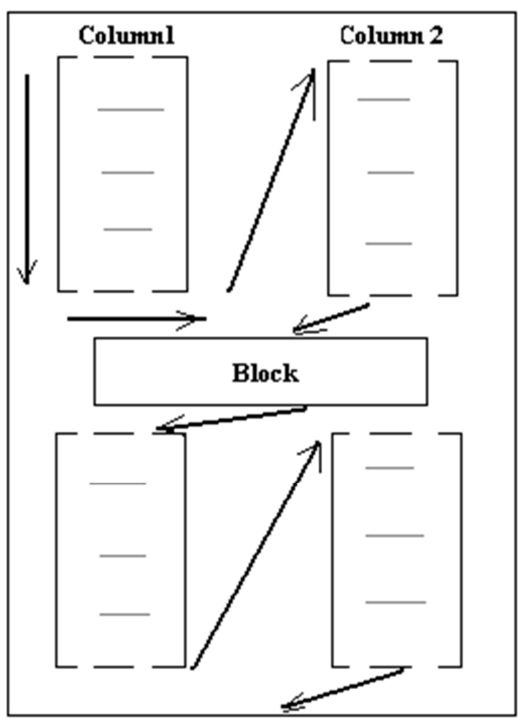

Figure 1. Method reading page with block.

\section{Acknowledgements}

The authors express their gratitude to Prof. Abdulla Y Al Hawaj, Founder of Ahlia University for all the support provided.

\section{References}

[1] Al Cheikha A. H. (2018; 2, 11), "Generating new binary orthogonal sequences using quotient rings $\mathrm{Z} / p^{m} \mathrm{Z}$ ", Research Journal of Mathematics and Computer Science (RJMCS), pp 113. 
[2] Al Cheikha A. H. (2016), "Compose Walsh's Sequences and MSequences", International journal of computer and technology (IJCT), Vol. 15, No. 7, 2016. pp. 6933- 6939.

[3] Byrnes, J. S., Swick, D. A. (1970), "Instant Walsh Functions", (SIAM Review., Vol. 12, pp.131.

[4] Yang S. C, (1998),"CDMA RF System Engineering," Boston, London Artech House.

[5] Thomson, J. T. (2013), “Abstract Algebra Theory and Applications," Free Software Foundation.

[6] Al Cheikha A. H. (2005), "Isomorphic Sequences Sets Generation of the Walsh Sequences", Qatar University Science Journal Vol. 25, 2005. pp. 16-30.

[7] Lidl, R., Pilz, G. (1984), "Applied Abstract Algebra”, New York Springer-Verlage New York.

[8] Mac Williams, F. G., Sloane, G. A. (2006), The Theory of ErrorCorrecting Codes. Amsterdam North-Holland.

[9] Lidl, R., Nidereiter, H. (1994), "Introduction to Finite Fields and Their Application," Cambridge University USA,.

[10] Sloane, N. J. A. (1076), "An Analysis Of The Stricture and Complexity Of Nonlinear Binary Sequence Generators," IEEE Trans. Information Theory Vol. It 22, No 6, PP 732- 736.

[11] Al Cheikha A. H., Ruchin J. (March, (2014), "Generation of Orthogonal Sequences by Walsh Sequences" International Journal of Soft Computing and Engineering Vol.4, Issue- 1, pp. 182-184.
[12] Jong, N. S., Golomb, S. W., Gong, G., Lee, H. K., Gaal, P. (1998), "Binary Pseudorandom Sequences For period $2^{\mathrm{n}}-1$ with Ideal Autocorrelation," IEEE Trans. Information Theory, Vol. 44 No 2, PP. 814-817.

[13] Lee, J. S., Miller, L. E. (1998), CDMA System Engineering Hand Book. Boston, London Artech House.

[14] Yang, K., Kim, Y. K., Kumar, P. V. (2000), "Quasi-orthogonal Sequences for code - Division Multiple Access Systems," IEEE Trans. information theory, Vol. 46 No3, Pp. 982- 993,.

[15] Al Cheikha A. H. (30th December 2015), "Compose Walsh's Sequences and Reed Solomon Sequences", ISERD International Conference, Cairo, Egypt, ISBN: 978-93- 8583290-1, pp. 23-26.

[16] Kacami, T., Tokora, H. (1978), "Teoria Kodirovania", MOSCOW: Mir.

[17] Al Cheikha A. H. (July, 2017). Compose M-Sequences. Australian Journal of Business, Social Science and Information Technology. AJBSSIT. Vol. 3, Issue 3. Pp. 119126. (Australia and New Zealand Business and Social Science Research Conference (ANZBSRC) 2016).

[18] Farleigh, J. B. (1971), A First course In Abstract Algebra, Fourth printing. Addison-Wesley publishing company USA.

[19] David, J. (2008), “Introductory Modern Algebra," Clark University USA. 